\title{
A New Scattering Clusters Equalization Algorithm for Airtarget Acoustic Passive Detection and Classification
}

\author{
Mazhar Tayel \\ EED, Faculty Of Engineering, \\ Alexandria University, \\ Alexandria, Egypt.
}

\author{
Mahmoud Sabry \\ EED, Faculty Of Engineering, \\ Alexandria University, \\ Alexandria, Egypt.
}

\begin{abstract}
Capture of airtarget acoustic signal carries a lot of information that can be used for its characterization. The airtarget acoustic signal can be used as a passive detection and classification technique. In this paper, a proposed flexible algorithm for airtarget type passive detection and classification is introduced to extract some selected unique features to classify airtargets. Also, a proposed equalization method is introduced to characterize the airtargets according to their extracted features.
\end{abstract}

\section{General Terms}

This research is concerned with introducing a proposed algorithm to classify airtarget acoustic signal using two methods of analysis. Moreover, an equalization method is introduced using the derived features to insure success of classification

\section{Keywords}

Airtarget, Acoustic signal, Short time series, Decomposition domain and Equalization analysis.

\section{INTRODUCTION}

Radar is an active technique for Airtarget (AT) detection. The Electronic Counter Measurement (ECM) system techniques are easily able to localize the AT and/not classify its type. However, detecting an AT passively is an attractive issue for Air defense systems. Also, Acoustic Signal (AS) can be used to introduce favorable information about ATs, especially, for high threats AT and small drones in very low altitude. In this paper, a proposed algorithm is presented to introduce a method for extracting some selected unique features able to deduce passively discrimination between ATs. Thus, features extraction operation from noisy AT is a challenge.

\section{METHODOLOGY}

Usually classification systems are based on a constructing learning library. A Vector Space Feature Library (VSFL) is constructed with a training algorithm. In the present work an algorithm is introduced using two different analysis approaches: a Short-time Series Analysis and a Hybrid decomposition domain Analysis [1], [2]. Also, a proposed equalization method is introduced to assess and discriminate featured parameters for classification process.

\section{THE PROPOSED CLASSIFICATION ALGORITHM}

The present proposed classification algorithm (PCA) illustrates a new concept to study and analyze AT-AS. The PCA consists mainly of four stages: Input stage,
Preconditioning stage, Processing stage and Classification stage, as shown in Figure (1).

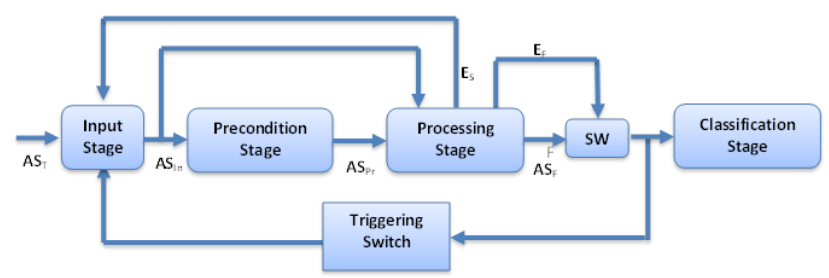

Fig.1 The basic concept block diagram of the PCA

The AT-AS is captured as $\mathrm{AS}_{\mathrm{T}}$ at the Input stage which consists of three blocks: a sensitive microphone, Instrumentation Amplifier, a Low Pass Filter and a Sampler. A signal $\mathrm{AS}_{\text {In }}$ derived from the Input stage is used to feed both a Preconditioning stage and a Processing stage simultaneously. The $\mathrm{AS}_{\text {In }}$ is to be converted and enhanced in the Preconditioning stage applying two processes: Analog-toDigital Converter and a proposed Signal Normalization. A second $\mathrm{AS}_{\mathrm{Pr}}$ derived from the Processing stage is directed to pass through two different paths channels: feature extraction channel and control channel. The AT-AS features are extracted in the Feature Channel of the Processing stage, obeying to internal decision coming from the control channel. The decision produces a control signal to switch the extracted features depending on the assessment test. These extracted features are simultaneously fed to the Classification stage and to a triggering switch to enable the Input stage to pass a next captured signal. The classification stage uses the extracted features to classify the ATs using a reference VSFL. The difference between the extracted features and that stored in the VSFL is used to determine the probability of the predicted AT type.

In this work, two proposed analysis methods: Normalization and Equalization are used in the processing stage to decompose the $\mathrm{AS}_{\mathrm{Pr}}$ into coefficients that is to be analyzed.

\subsection{The Frist Proposal: A Time Domain Analysis Method}

The first proposal method follows the PCA given in figure (1). It is a time series analysis using short intervals of AT-AS to be processed in the time domain and to deduce selected unique features assisting in the classification process. Figure (2) shows the block diagram of the short time series analysis method. It has two main channels namely: Feature Channel and Control Channel. The feature channel is used to analyze the preconditioned signal $\mathrm{AS}_{\mathrm{Pr}}$ producing unique features for AT-AS discrimination. In the introduced analysis, the short length series downloaded files. 


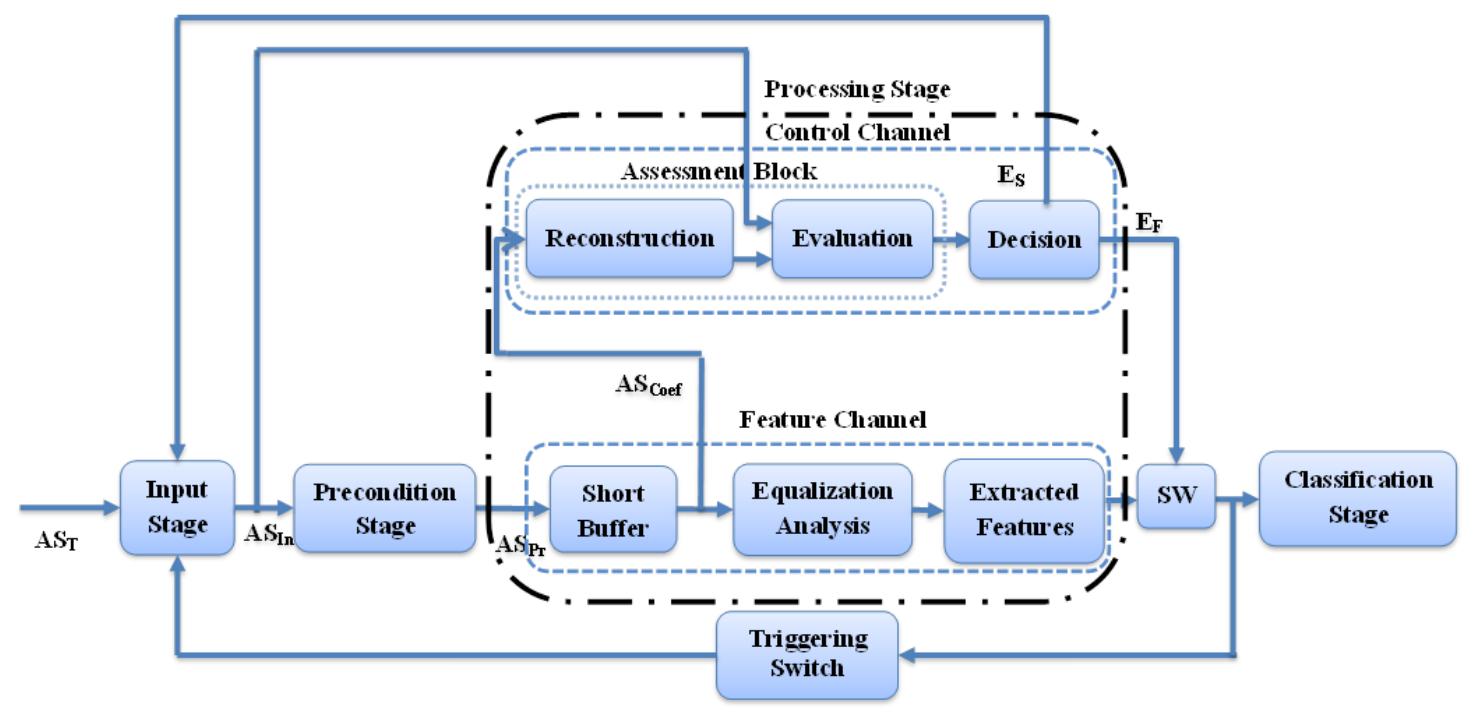

Fig.2 The functional block diagram for the short time series analysis

\subsection{The Second Proposal: A Hybrid Domains Analysis Method}

The second proposal method depends mainly on Discrete Wavelet Transform (DWT) analysis for feature extraction considering its powerful filtration property [3], [4] and representing the AT-AS in the decomposition domain. This hybrid domains method has three main channels in the modified processing stage, namely: Down Sampling Channel, Feature Channel and Control Channel. The Down Sampling Channel uses the Discrete Cosine Transform (DCT) to calculate a stopping level to produce a specified operating Bandwidth (BW) for the DWT processing in the Feature Channel, because the produced approximation from the DWT processing on the tested AT-AS does not saturated as in image processing to deep levels. The control channel produces enable and disable signals for different parts in the proposed method. The functional block diagram of the hybrid domains method processing stage is shown in figure (3). The modified processing stage is tested to extract the AT-AS features using histogram and equalization analyses in the Time-Frequency domain.

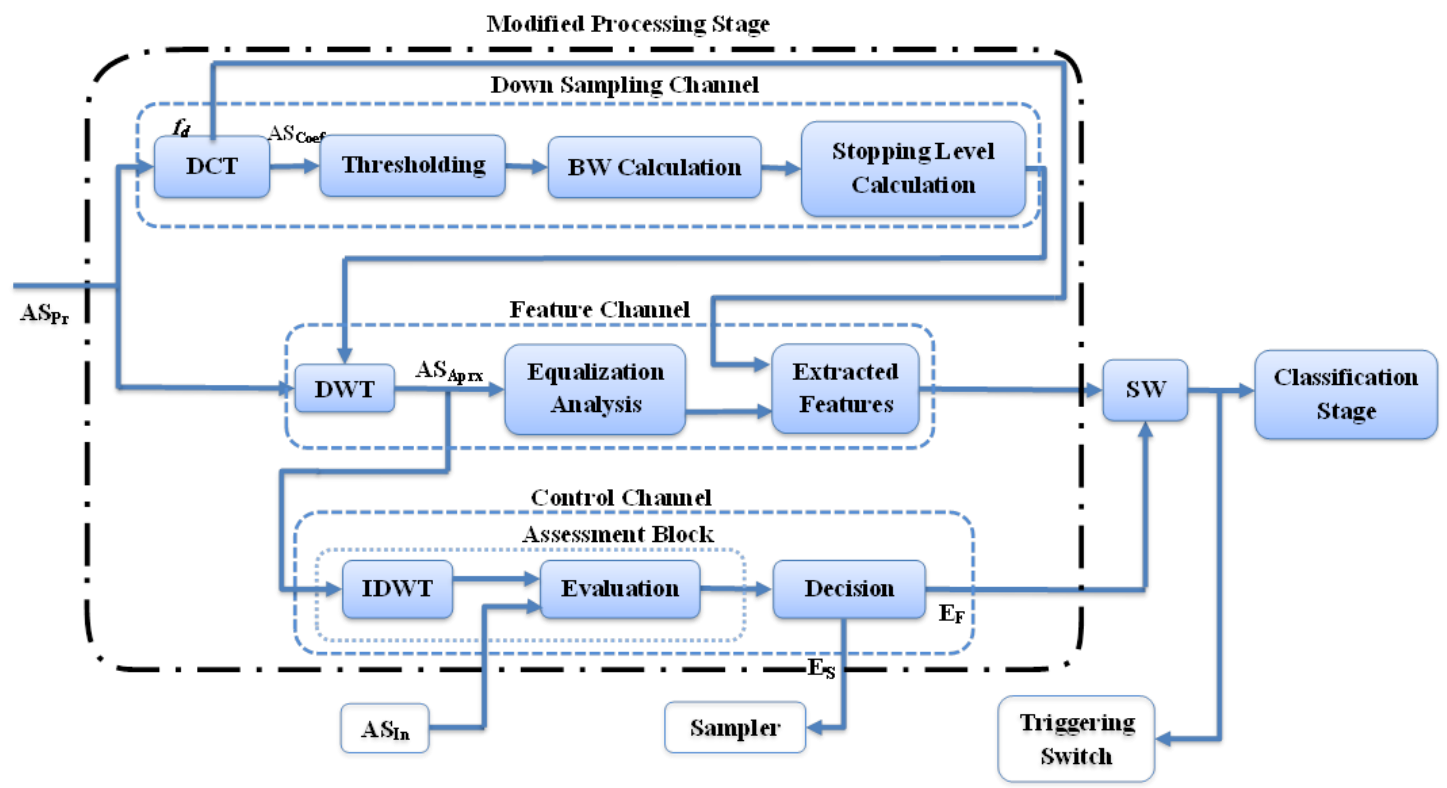

Fig.3 The functional block diagram of the Modified Processing Stage

\section{THE PROPOSED EQUALIZATION METHOD}

Histogram analysis has wide application in image processing, studying the recurrent intensity distribution of different colors [5]. So, it can be applied to study the AS in time domain describing the signal amplitude in a density function distribution. It divides the amplitude into equal Bins and counts the located samples in the Bins-Amplitude range. Therefore, the equalization analysis in this application is different than image processing.
In this research, the equalization is used to find a Normalized quantity in a percentage representation for the counted samples in histogram Bins offering a fair comparison between different lengths and sampling rate for different simulated AT-AS. It is calculated as:

\section{Peak Percentage No of samples in bin \\ $=\frac{\text { Total No.of samples in the file }}{}$}




\section{SIMULATION AND RESULTS}

The PCA is tested using the two proposed analysis methods for different types of AT downloaded AS [6], [7]. Six AT (3 Jets and 3 Propellers) ASs are selected to test the PCA. The tested signals from the downloaded files are 2-9 Seconds in length. The simulation is implemented using MATLAB programming to extract the selected unique features statistically. The proposed analysis methods use the ShortTime Series analysis and the hybrid domains analysis.

\subsection{Equalization Analysis in Time Domain}

The downloaded AT-AS files are treated as Short-Time Series signal to be analyzed. The analysis is used to check the capability of the feature channel in the processing stage to extract selected unique features to classify the AT-AS using equalization analysis. The histogram analysis is applied to the tested AT-AS files. Different parameters are calculated and equalization method is performed as listed in table (1). The AT-AS equalization analysis is shown in figure (4).

Table 1. The histogram and equalization parameters for different ATs in the Time Domain

\begin{tabular}{|c|c|c|c|c|c|c|c|c|c|}
\hline \multirow[b]{2}{*}{ No } & \multicolumn{2}{|c|}{ Airtarget } & \multicolumn{3}{|c|}{ File Analysis Parameters } & \multicolumn{2}{|c|}{$\begin{array}{l}\text { Histogram } \\
\text { Parameters }\end{array}$} & \multicolumn{2}{|c|}{$\begin{array}{c}\text { Equalization } \\
\text { Parameters }\end{array}$} \\
\hline & Engine & Type & $\begin{array}{c}\text { File } \\
\text { length } \\
(\mathrm{Sec})\end{array}$ & $\begin{array}{c}\text { Sampling } \\
\text { frequency } \\
(\mathrm{Hz})\end{array}$ & $\begin{array}{c}\text { No. of } \\
\text { Samples }\end{array}$ & $\begin{array}{l}\text { Min } \\
\text { counts }\end{array}$ & $\begin{array}{l}\text { Max } \\
\text { counts }\end{array}$ & $\begin{array}{c}\text { Max } \\
\text { counts } \\
{[\%]}\end{array}$ & $\begin{array}{c}\text { Min } \\
\text { counts } \\
{[\%]}\end{array}$ \\
\hline 1 & \multirow{3}{*}{ Jet } & Boing-707 & 9.67 & 8000 & 77366 & 9 & 28413 & 36.73 & 0.01 \\
\hline 2 & & F-15 & 2.26 & 8000 & 18103 & 45 & 6482 & 35.81 & 0.25 \\
\hline 3 & & F-16 & 4.76 & 11025 & 52440 & 48 & 20217 & 38.55 & 0.09 \\
\hline 4 & \multirow{3}{*}{ Propeller } & $\begin{array}{l}\text { B-131 } \\
\end{array}$ & 4.28 & 11025 & 47191 & 4 & 7366 & 15.61 & 0.008 \\
\hline 5 & & B-25 & 8.51 & 11025 & 93828 & 261 & 13844 & 14.75 & 0.27 \\
\hline 6 & & Quad Copter & 8.02 & 44100 & 353682 & 11 & 91946 & 25.99 & 0.03 \\
\hline
\end{tabular}

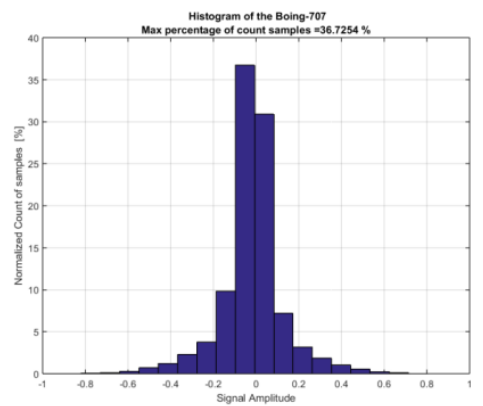

a): Equalization of Boing-707

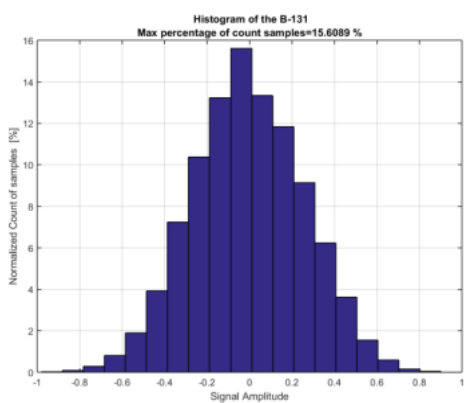

d): Equalization analysis of B-131

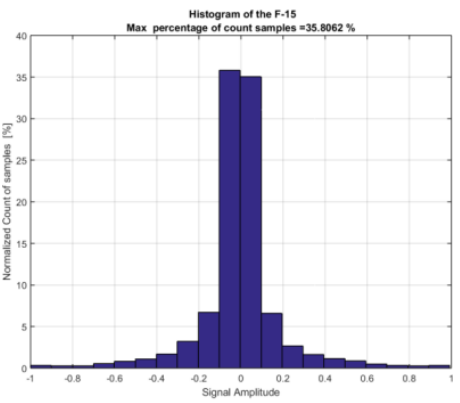

b): Equalization analysis of F-15

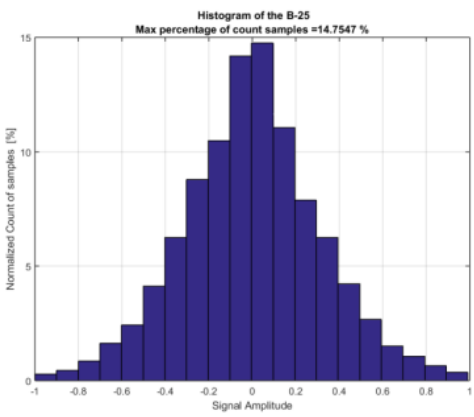

e): Equalization analysis of B-25

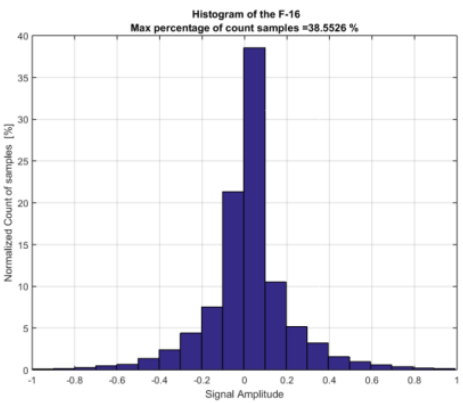

c): Equalization analysis of F-16

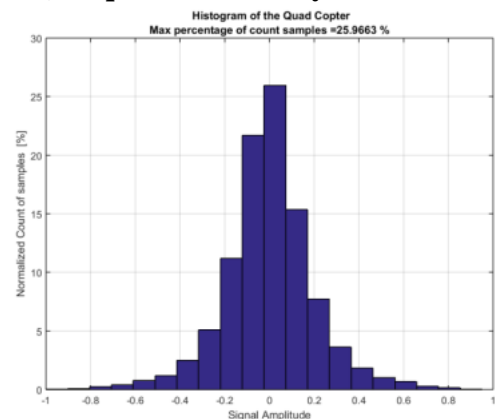

f): Equalization analysis of Quad Copter

Fig. 4 The equalization analysis of different AT-AS in the time domain

\subsubsection{Equalization Statistical parameters}

The Statistical parameters are calculated to extract discriminated features characterizing the AT-AS. The calculated parameters are: RMS, Variance, Skewness, and kurtosis. The calculated parameters are listed in table (2).

Table 2. The Powers of equalization parameters for different ATs using the time domain method

\begin{tabular}{|c|c|c|c|c|c|c|}
\hline \multirow{2}{*}{ No } & \multicolumn{2}{|c|}{ Aircraft } & \multicolumn{3}{c|}{ Power for equalization parameters } \\
\cline { 2 - 7 } & Eng. & Type & RMS & Variance & Skewness & Kurtosis \\
\hline 1 & $\stackrel{\Xi}{\leftrightarrows}$ & $\begin{array}{c}\text { Boing- } \\
\mathbf{7 0 7}\end{array}$ & 11.2 & 104.54 & 2405 & 73388 \\
\hline
\end{tabular}

\begin{tabular}{|c|c|c|c|c|c|c|}
\hline 2 & & F-15 & 11.5 & 111.85 & 2759 & 85966 \\
\hline 3 & & F-16 & 10.4 & 88.20 & 2059 & 67179 \\
\hline 4 & \multirow{3}{*}{ 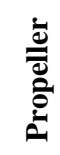 } & B-131 & 7.3 & 29.21 & 98 & 1492 \\
\hline 5 & & B-25 & 6.8 & 22.36 & 77 & 1065 \\
\hline 6 & & $\begin{array}{l}\text { Quad } \\
\text { copter }\end{array}$ & 7.9 & 40.14 & 229 & 3552 \\
\hline
\end{tabular}

The scattering plot is used as a new illustrative clustering method to deduce some selected unique features for the ATAS as shown in figure (5). Figure (5) shows the different moment orders against max peak percentage of equalization. 


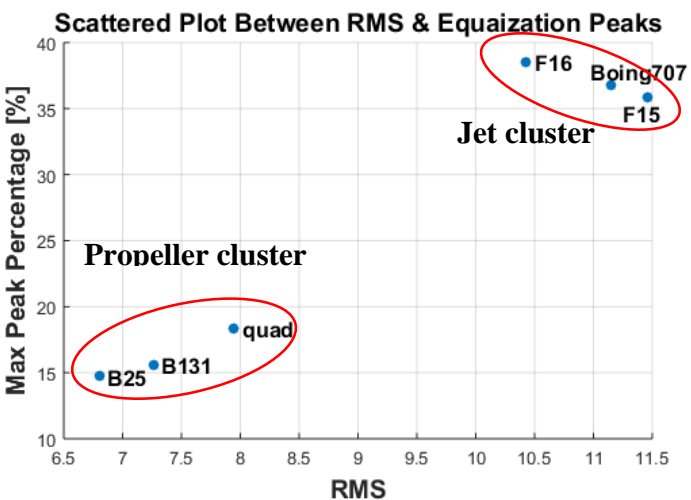

a): RMS-Equalization peaks scattering clusters

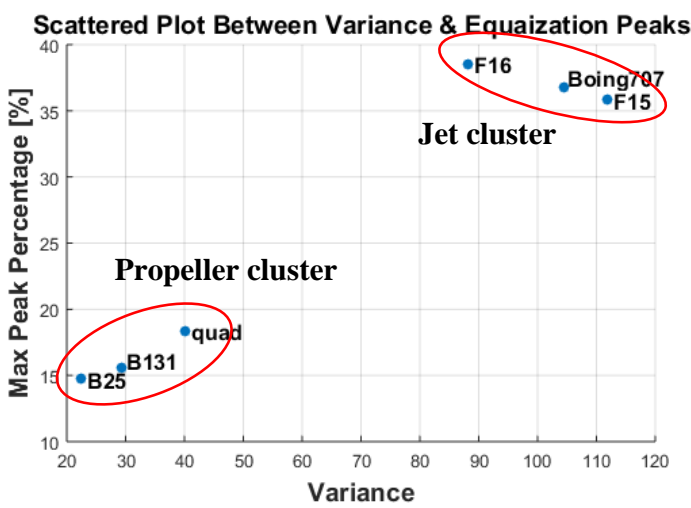

b): Variance-Equalization peaks scattering clusters

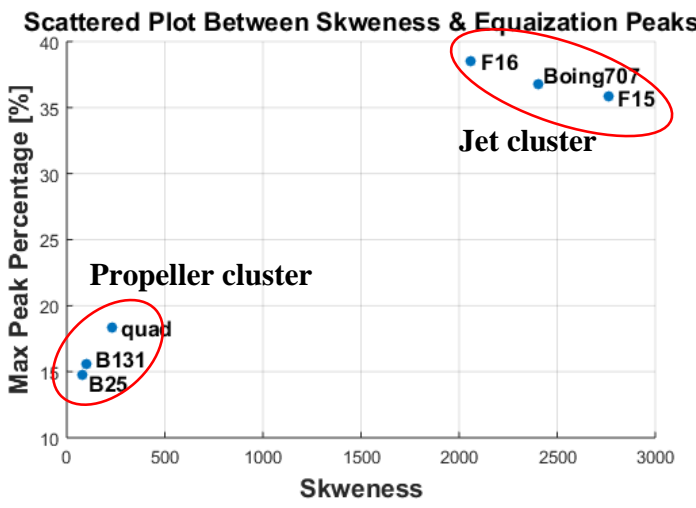

c): Skewness-Equalization scattering clusters

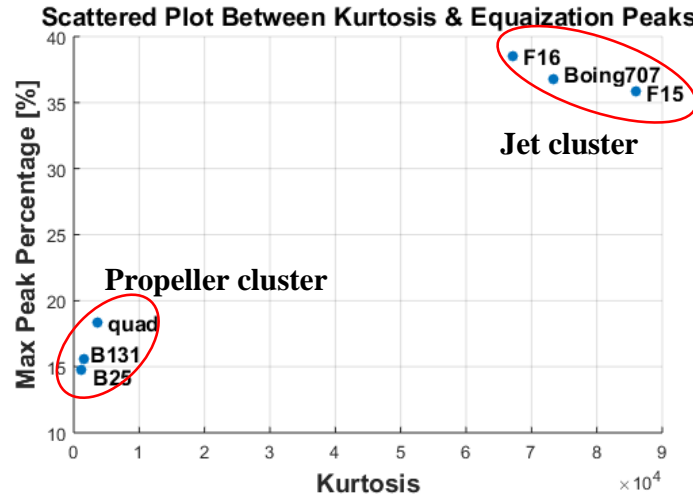

d): Kurtosis-Equalization scattering clusters

Fig.5 The scattering clusters for different statistical parameters in the time domain

From figure (5), it is quite evident the presence of classification clusters among the jet ATs and propeller ATs. Moreover, inside the same class clusters, there are discrimination among the tested ATs

\subsection{Equalization Analysis in Decomposition Domain}

The same downloaded files have been used to test the proposed hybrid domains method. The equalization is used to extract the AT-AS features. The equalization analysis is applied to the derived approximation coefficients of the tested AT-AS files at the calculated stopping level $\left(\mathrm{L}_{\mathrm{s}}\right)$ of The DWT processing in the decomposition domain. The Different parameters are calculated and presented in table (3). The equalization analysis for different AT-AS is shown in figure (6).

\subsubsection{Equalization Statistical parameters}

The Statistical parameters are calculated to get more discriminated features characterizing the AT-AS in the decomposition domain. The first four parameters are calculated: RMS, Variance, Skewness and kurtosis. The calculated parameters are listed in table (4).

Table 3. The histogram and equalization parameters for different ATs in the hybrid domains method

\begin{tabular}{|c|c|c|c|c|c|c|c|c|c|}
\hline \multirow[b]{2}{*}{ No } & \multicolumn{2}{|c|}{ Airtarget } & \multicolumn{3}{|c|}{ Hybrid Analysis Parameters } & \multicolumn{2}{|c|}{$\begin{array}{l}\text { Histogram } \\
\text { Parameters }\end{array}$} & \multicolumn{2}{|c|}{$\begin{array}{c}\text { Equalization } \\
\text { Parameters }\end{array}$} \\
\hline & Engine & Type & $\begin{array}{c}\text { Sampling } \\
\text { frequency } \\
(\mathbf{H z})\end{array}$ & $\begin{array}{c}\text { Stopping } \\
\text { Level }\end{array}$ & $\begin{array}{c}\text { Level } \\
\text { Samples }\end{array}$ & $\begin{array}{l}\text { Min } \\
\text { counts }\end{array}$ & $\begin{array}{l}\text { Max } \\
\text { counts }\end{array}$ & $\begin{array}{c}\text { Max } \\
\text { counts } \\
{[\%]}\end{array}$ & $\begin{array}{c}\text { Min } \\
\text { counts } \\
{[\%]}\end{array}$ \\
\hline 1 & \multirow{3}{*}{ Jet } & Boing-707 & 8000 & 4 & 4847 & 0 & 2445 & 50.44 & 0.00 \\
\hline 2 & & F-15 & 8000 & 5 & 578 & 1 & 210 & 36.33 & 0.17 \\
\hline 3 & & F-16 & 11025 & 5 & 1651 & 1 & 817 & 49.49 & 0.06 \\
\hline 4 & \multirow{3}{*}{ Propeller } & B-131 & 11025 & 5 & 1487 & 1 & 167 & 11.23 & 0.07 \\
\hline 5 & & B-25 & 11025 & 5 & 2944 & 2 & 577 & 19.60 & 0.07 \\
\hline 6 & & Quad Copter & 44100 & 8 & 1394 & 2 & 229 & 16.43 & 0.14 \\
\hline
\end{tabular}




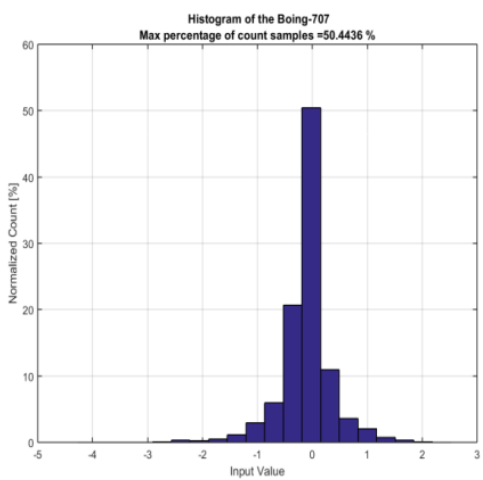

a): Equalization analysis of Boing-707

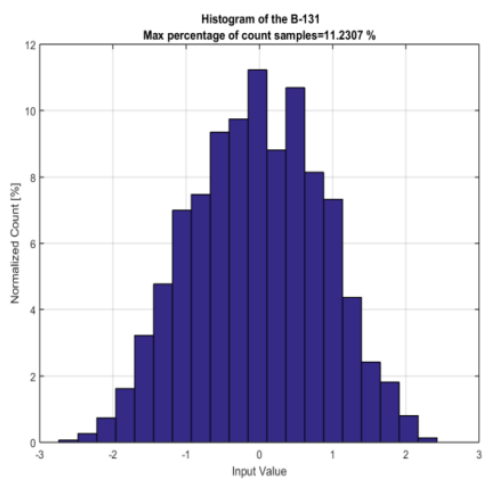

d): Equalization analysis of B-131

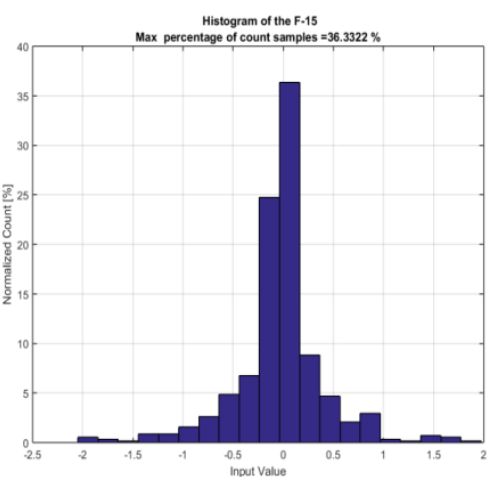

b): Equalization analysis of F-15

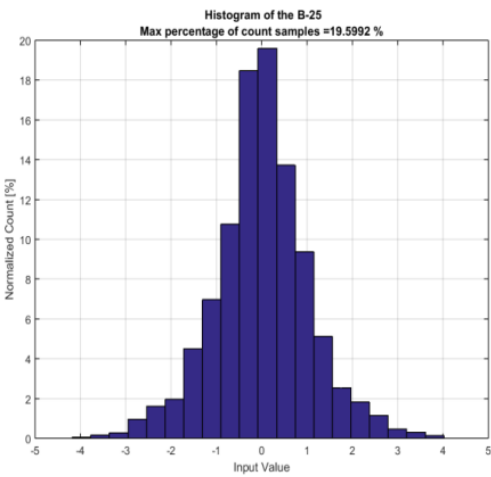

e): Equalization analysis of B-25

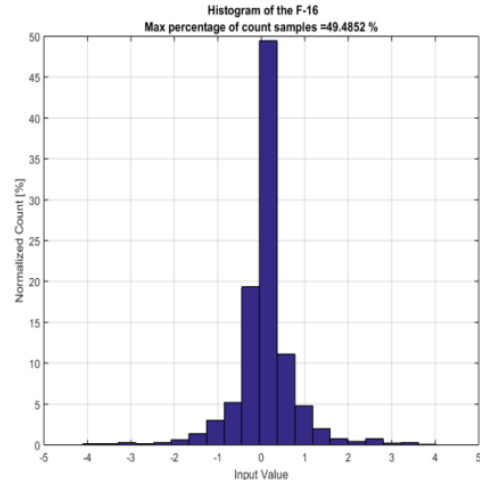

c): Equalization analysis of F-16

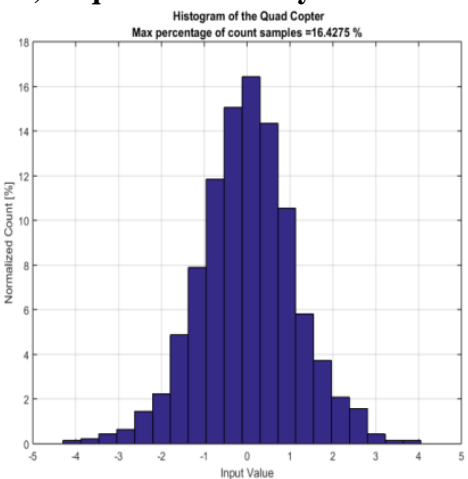

f): Equalization analysis of Quad Copter

Fig.6 The equalization analysis for different AT-AS in the hybrid domains method

Table 4. The Powers of equalization parameters for different ATs in the hybrid domains method

\begin{tabular}{|c|c|c|c|c|c|c|}
\hline \multirow{2}{*}{ No } & \multicolumn{2}{|c|}{ Aircraft } & \multicolumn{4}{|c|}{ Power for equalization parameters } \\
\hline & Eng. & Type & RMS & Variance & Skewness & Kurtosis \\
\hline \multirow{3}{*}{$\begin{array}{l}1 \\
2 \\
3\end{array}$} & \multirow{3}{*}{$\stackrel{\Xi}{\circ}$} & $\begin{array}{c}\text { Boing- } \\
707\end{array}$ & 12.5 & 139.8 & 4824.67 & 216689 \\
\hline & & F-15 & 10.3 & 85.56 & 1874.20 & 56020 \\
\hline & & F-16 & 12.2 & 132.1 & 4493.72 & 198285 \\
\hline 4 & \multirow{3}{*}{ 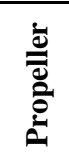 } & B-131 & 6.2 & 15.28 & 7.56 & 320 \\
\hline 5 & & B-25 & 7.8 & 38.50 & 281.02 & 4482 \\
\hline 6 & & $\begin{array}{c}\text { Quad } \\
\text { copter }\end{array}$ & 7.4 & 31.71 & 149.14 & 2100 \\
\hline
\end{tabular}

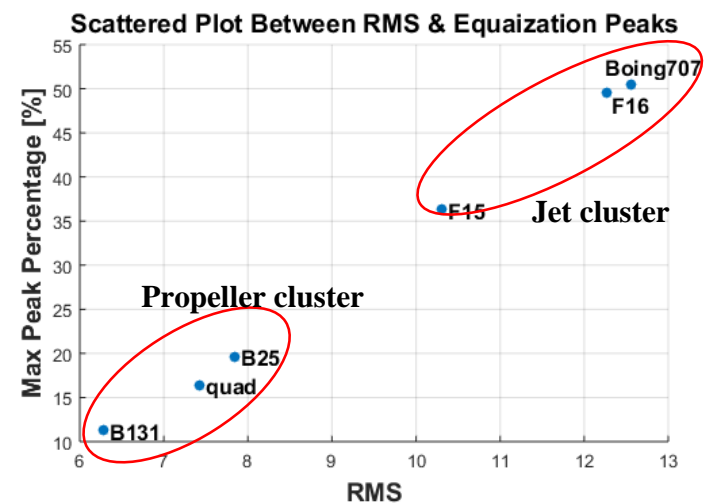

a): RMS-Equalization peaks scattering clusters

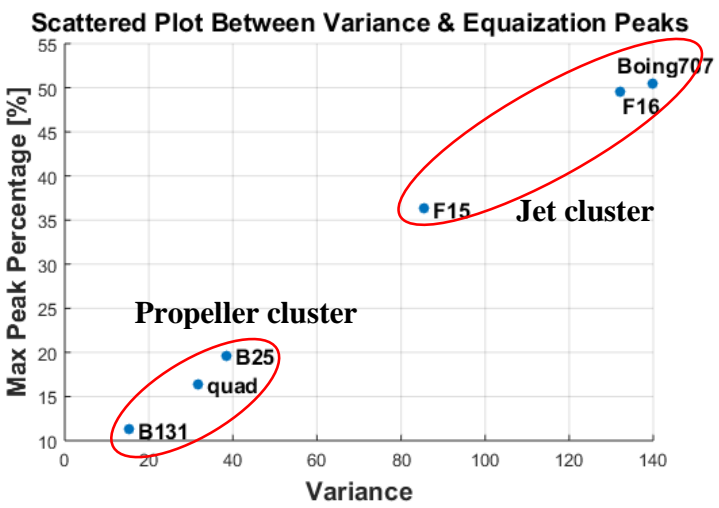

b): Variance-Equalization peaks scattering clusters
The scattering plot is used to deduce the selected unique features for the AT-AS using the decomposition method as shown in figure (7). Figure (7) shows the different moment orders against the equalization parameters. It is worth to note that, the classification lobs are well discriminated. Also, inside the same class lobe, there is discrimination among the tested ATs. 


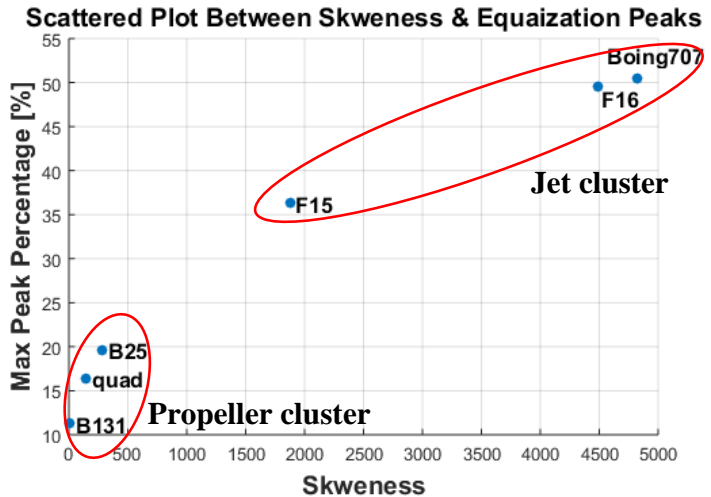

c): Skewness \& Equalization peaks scattering clusters

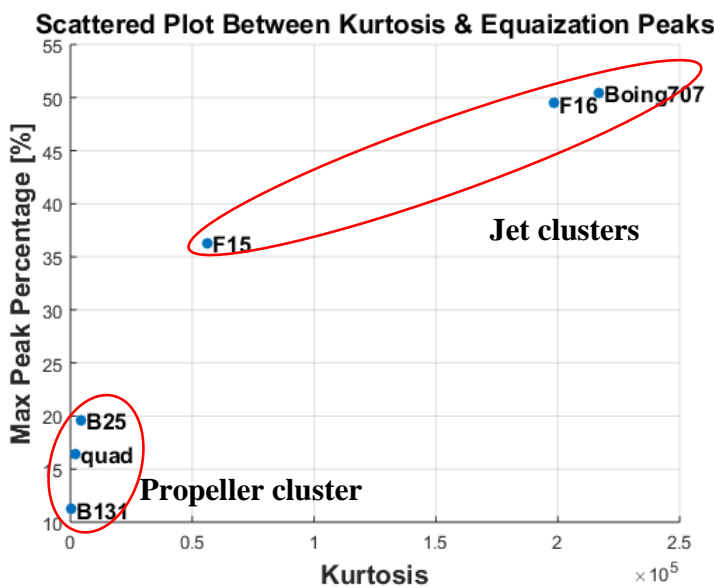

d): Kurtosis-Equalization peaks scattering clusters

Fig.7 The scattering clusters for different statistical parameters in the hybrid domains method

\section{CONCLUSION}

The application of the PCA to test AT samples shows existence of discriminated class clusters, that can be applied for passive detection and discrimination among the AT types. The introduced PCA for passive detection and discrimination provides more flexibility to work in different analysis domains. It is able to derive many unique features about the AT-AS to be characterized. The two analysis methods can be used to verify the provided extracted features. However, the time domain analysis is recommended to be used for fast analysis depending on its simplicity. The hybrid domains analysis method is recommended to be used in the noisy environmental conditions, verifying the results of the short time series method. The normalization, decomposition and equalization analyses method proved that it is able to offer a fair comparison values in the classification process even the length of the captured AT-AS is varying. These provided results from the proposed methods will be used to build a neural network classifier to discriminate among ATs with low rate of false alarm

\section{REFERENCES}

[1] Mazhar Tayel and Mahmoud Sabry. 2016. A Detection and Identification Method for Airtarget Acoustic Signal Characterization. IOSR Journal of VLSI and Signal Processing (IOSR-JVSP) Volume 6, Issue 2, Ver. I (Mar. -Apr. 2016), PP 21-24 e-ISSN: 2319 - 4200, p-ISSN No. : $2319-4197$.

[2] Mazhar Tayel and Mahmoud Sabry. 2016. A Proposed Hybrid Method for Airtarget Acoustic Signature Diagnosis. International Journal of Science and Research (IJSR) 2319-7064.

[3] Anamitra Bardhan Roy. 2012. Comparison of FFT, DCT, DWT, WHT Compression Techniques on Electrocardiogram. Special Issue of International Journal of Computer Applications 0975 - 8887.

[4] Erik Axel Rønnevig Nielsen and DR. Anne Cathrine Elster 2007. Real-Time Wavelet Filtering on the GPU. Norwegian University of Science and Technology Thesis. May 2007.

[5] Singh RP, Dixit M. 2015. Histogram Equalization Techniques for Image Enhancement .International Journal of Signal Processing, Image Processing and Pattern Recognition 345-52.

[6] Airplane Sounds, Jet Sounds. Available. 2017. http://www.partnersinrhyme.com/soundfx/airplanesoundf x.shtml.

[7] Airplane Sounds, Jet Sounds. Available. 2017. http://www.aviationtrivia.info/AIRCRAFT-WAVSOUNDS.php. 